\title{
The MU-RAY project: Summary of the round-table discussions
}

\author{
F. Beauducel ${ }^{1}$, A. Bross ${ }^{2}$, S. Buontempo ${ }^{3}$, L. D’Auria ${ }^{4}$, Y. Déclais ${ }^{5}$, G. De Lellis ${ }^{3,6}$, G. Festa ${ }^{6}$, P. Gasparini' ${ }^{6,7}$, D. Gibert ${ }^{1}$, \\ K. Hoshina ${ }^{8}$, G. Iacobucci ${ }^{3}$, N. Lesparre ${ }^{1}$, G. Macedonio ${ }^{4}$, A. Marotta ${ }^{3}$, J. Marteau ${ }^{5}$, M. Martini ${ }^{4}$, G. Miele ${ }^{3,6}$, \\ P. Migliozzi ${ }^{3}$, C. A. Moura ${ }^{3}$, M. Orazi ${ }^{4}$, A. Pla-Dalmau ${ }^{2}$, O. Pisanti ${ }^{3,6}$, S. Pastor ${ }^{9}$, R. Peluso ${ }^{4}$, \\ P. Rubinov ${ }^{2}$, G. Scarpato ${ }^{4}$, G. Sekhniaidze ${ }^{3}$, P. Strolin ${ }^{3,6}$, H. Taira ${ }^{10}$, M. Tanaka ${ }^{11}$, \\ H. K. M. Tanaka ${ }^{10}$, A. Tarantola ${ }^{1}$, T. Uchida ${ }^{12}$, M. Vassallo ${ }^{3,7}$, \\ I. Yokoyama ${ }^{13}$, and A. Zollo ${ }^{6}$ \\ ${ }^{1}$ Institut de Physique du Globe, Paris, (IPGP), France \\ ${ }^{2}$ Fermi National Accelerator Laboratory (FNAL), Batavia, IL, USA \\ ${ }^{3}$ Istituto Nazionale di Fisica Nucleare (INFN), Sezione di Napoli, Italy \\ ${ }^{4}$ Osservatorio Vesuviano, INGV, $(\mathrm{OV})$, Italy \\ ${ }^{5}$ Institut de Physique Nucléaire de Lyon (IPNL), France \\ ${ }^{6}$ Università di Napoli "Federico II" (UniNA), Italy \\ ${ }^{7}$ Consorzio Analisi e Monitoraggio Rischi Ambientali (AMRA), Napoli, Italy \\ ${ }^{8}$ University of Wisconsin, Madison, USA \\ ${ }^{9}$ Instituto de Física Corpuscular (CSIC-Universitat de València), Spain \\ ${ }^{10}$ Earthquake Research Institute (ERI), University of Tokyo, Japan \\ ${ }^{11}$ Institute of Particle and Nuclear Studies, KEK, Japan \\ ${ }^{12}$ Department of Physics, University of Tokyo, Japan \\ ${ }^{13}$ Usu Volcano Laboratory, Hokkaido University, Japan
}

(Received December 3, 2008; Revised March 3, 2009; Accepted March 30, 2009; Online published February 22, 2010)

The MU-RAY project has the challenging aim of performing muon radiography of the summit cone of Mt. Vesuvius. The muon telescopes developed for this purpose will be available for the radiography of other volcanoes, in particular Stromboli. The scientific goals, the strategy for their implementation and the baseline detector design are discussed in detail. A tentative time schedule for the project is drawn.

Key words: Muon, radiography, volcanoes.

\section{Introduction}

The MUon-RAdiographY (MU-RAY) project aims at performing muon radiographies of the internal structures of volcano edifices with higher sensitivity than so far achieved, by developing and exploiting a new generation of muon "telescopes". The first challenging aim is to perform the radiography of the summit cone of Mt. Vesuvius. The muon telescope will be available for the radiography of other volcanoes, in particular Stromboli. The methodology, established and successfully applied to several volcanoes in Japan (Tanaka et al., 2005, 2007a-c, 2008; Tanaka and Yokoyama, 2008) relies on the observation of the absorption by the rock of quasi-horizontal muons produced by the decay of secondary particles in the cascades originated by the interaction of the primary cosmic radiation with the earth atmosphere. So far, angular resolutions down to $30 \mathrm{mrad}$ were obtained with this method, corresponding to spatial resolutions of 30 meters for a detector positioned at $1 \mathrm{~km}$ from the center of the volcano.

Mt. Vesuvius is located $15 \mathrm{~km}$ east of the city of Naples. Its morphology is characterized by a volcanic cone (called

Copyright (C) The Society of Geomagnetism and Earth, Planetary and Space Sciences (SGEPSS); The Seismological Society of Japan; The Volcanological Society of Japan; The Geodetic Society of Japan; The Japanese Society for Planetary Sciences; TERRAPUB.

doi:10.5047/eps.2009.03.004
Gran Cono), which reaches $1280 \mathrm{~m}$ a.s.l., standing in the caldera of Mt. Somma. Over the years, this volcano went through a series of violent and destructive eruptions. That of AD 79 destroyed Pompeii, Herculaneum and Stabiae. The last period of persistent volcanism of Mt. Vesuvius started after the 1631 Plinian eruption and lasted till 1944. Since that time Mt. Vesuvius has been quiescent and actually only a moderate seismicity and fumaroles testify its activity. The chronology suggests that the longer is the quiescence period the more violent is the renewal of activity. Mt. Vesuvius represents the highest volcanic risk in Europe, since about 600,000 people are living in the "red" zone around it. For this reason, a strong social motivation to this enterprise fosters the scientific interest.

The volcano has been the object, in recent years, of thorough geophysical and volcanological studies (Zollo et al., 1996, 2002; Auger et al., 2001). The volcanic activity of Mt. Vesuvius is monitored by a multi-parametric observation system with continuous recording of seismicity, ground deformation and gas emission data that are transmitted to the operating centre of the Vesuvian Observatory, located downtown in Naples. This careful monitoring of Mt. Vesuvius has the purpose to allow predicting when the next eruption will happen, with enough forewarning to be able to safely evacuate the most risky areas. The detailed scientific studies performed over the years, allowed to establish 
a model for the volcano down to depths of several $\mathrm{km}$ with precisions of several hundreds meters. To obtain a more trustful model of the volcano and in particular to gather information on the dynamics of the next eruption, i.e. how next eruption will develop, these results should be complemented with those coming from muon radiography, which are much more precise although relative only to the topmost few hundreds meters. As an example, the mass ejection rate during an eruption depends very strongly on the diameter of the lava conduit: the presence of a lava conduit ranging in diameter from $50 \mathrm{~m}$ to $100 \mathrm{~m}$ would produce an increase by an order of magnitude of the mass ejection rate during an eruption (see the contribution by G. Macedonio to this workshop). Therefore, the knowledge of the lava conduit dimensions and shape with a resolution of the order of $10 \mathrm{~m}$ will be beneficial in forecasting the dynamics and the effects of the next eruption.

Given all the above arguments and motivations, the primary goal of the MU-RAY project is the challenging task of high-resolution radiography of Mt. Vesuvius. The measurements at Mt. Vesuvius are intended to be the first of a series of volcano radiographies around the world, that of Stromboli among them. Therefore, the project aims at collecting and focusing the international scientific interests for this new methodology and setting up the standards for a future generation of high-resolution detectors for muon radiography of volcanoes and other geological structures.

\section{Experimental Conditions and Strategy}

Up to now, the topmost internal structure of a volcano edifice was measured by conventional geological methods (seismological, gravimetric and electromagnetic), which provide spatial resolutions of the order of hundreds of meters. The detection of variations of the rock density at shallow depths, like those due to lava conduits or dykes, calls for an improvement of the measurement resolution by one order of magnitude. This task can be achieved by muon radiography. Structures of $10 \mathrm{~m}$ in size measured by a detector positioned at $1 \mathrm{~km}$ correspond to the measurement of $10 \mathrm{mrad}$ angles. In principle, such a precision is easily obtained by charged-particle detector technologies as developed in recent years for high-energy physics experiments. However, there are several limiting factors and experimental conditions that should be taken into account when planning the muon radiography of a volcano. Among them, the most relevant are:

- The muon absorption in 1.5-2 km of rock is quite severe; careful measurements are needed to tune a full Monte Carlo simulation.

- The multiple scattering in the rock leads to an angular uncertainty of about $10 \mathrm{mrad}$ on the average and larger for low momenta of the muons emerging from the mountain. The amount of this effect, which deteriorates the measurement resolution, and its details should be carefully evaluated and accounted for in the design of the detector.

- Volcanic environments are variable and harsh, presenting quite large temperature excursion. The ideal detector locations are often difficult to reach and sometimes the help of a helicopter is needed to transport heavy instruments. In normal cases, power lines are not be available.

In the case of Mt. Vesuvius, we should add to the above limiting factors the fact that the deep crater $(\sim 250 \mathrm{~m})$ and the mountain topology allows us to detect only those very energetic muons that were able to cross $2 \mathrm{~km}$ of rock. Therefore we expect a very low muon flux, which makes the muon radiography of Mt. Vesuvius a challenging task.

As a consequence of all these considerations, the following strategy has been set up for MU-RAY.

\subsection{Preliminary measurements}

Preliminary muon radiographies will be performed at Mt. Vesuvius in 2009. A muon telescope presently taking data in Japan will be used for this purpose. That telescope has an active area of $1 \mathrm{~m}^{2}$ and provides an angular resolution of $40 \mathrm{mrad}$ if the two planes of scintillating strips are positioned at a distance of $2 \mathrm{~m}$. These measurements will provide experimental data on the cosmic-ray flux through the Mt. Vesuvius edifice, which will be of primary importance in the design of the MU-RAY detector and in the planning of the data taking periods. Given the difficulty of simulating the behaviour of muons through large depths of rock, the measurements will have the important role of tuning the Monte Carlo simulation techniques. These first measurements are also intended to prepare the field for highresolution radiographies by experiencing the experimental and technical difficulties.

In the framework of the DIAPHANE project (Gibert et al., 2010), a conceptually similar detector, but of larger angular acceptance and improved spatial resolution, will be built to take data at La Soufriere in Guadalupe.

\subsection{Simulation strategy and Monte Carlo validation}

The simulation of the stochastic behaviour of very highenergy muons traversing large depths of rock is a complex task. A few simulation packages that, among other applications, can be used for this purpose are available. None of them is considered fully reliable by the astro-particle physics community. We will use both GEANT (GEANT4) and FLUKA (Ferrari et al., 2005) tracking programs, which are conceived for a full simulation of the interactions of particles with matter. A digital elevation map (DEM) of Mt. Vesuvius with the precision of $20 \mathrm{~m}$ and possibly better will be used to establish the amount of rock crossed by the muons. In parallel, an effort is going on that aims at applying at muon radiography the code written by some of us (Miele et al., 2006; Buontempo et al., 2010) to simulate the interaction of neutrinos with the Earth in the framework of the research program of Earth radiography using neutrinos. The contribution by E. Borriello et al. at this workshop gives more details on the Monte Carlo simulations.

The tuning and final validation of the Monte Carlo simulations will be obtained by comparison to the data taken at Mt. Vesuvius during 2009.

\subsection{Detector specifications and requirements}

Given the need to take data in areas of difficult accessibility, the detector must be modular, each module being compact and lightweight enough to be transported in the experimental area by humans or with the help of a small 
helicopter. The mechanical design should be such that the detector can be quickly assembled in situ and rugged enough to avoid damages while transporting or assembling it. The detector should require minimal maintenance, have low-power consumption and the least possible impact on the environment. Throughout the course of the data taking periods, expected to last several months, the personnel that controls the experimental area should be required to be on site only for limited time. Given the large amount of rock to be crossed, the muon flux is expected to drop drastically with respect to that measured by muon radiographies in Japan. Therefore, a detector active area of the order of $10 \mathrm{~m}^{2}$ or more is expected to be required to allow collection of enough statistics during data taking periods of several months. Finally, data should be immediately transferred to laboratories where the detector calibration and data quality could be checked in real time and the data analysis be performed.

\subsection{Construction and operation of the MU-RAY tele- scopes}

Three high-resolution muon detectors having each an area of $2 \times 2 \mathrm{~m}^{2}$ will be built for the challenging goal of the radiography of Mt. Vesuvius. The design will start soon and will comply with all the arguments listed above. It is foreseen that the telescopes could be located either close to each other to reduce the exposure time, or they could take data in different locations around the volcano for a 3-dimensional data taking (tomography). These detectors are intended as a standard for a future production that would allow the study of other volcanoes, in particular Stromboli.

\section{Detector Technologies}

Several detector technologies were considered. Among them plastic scintillators, nuclear emulsions and Resistive Plate Chambers (RPC) were carefully reviewed. In the case of nuclear emulsions, although they offer a very high resolution and have no need for electrical power, they do not allow real-time data acquisition and transfer to laboratories for data analysis. In the case of RPCs, the sensitivity to temperature, the need for a gas mixture to be kept carefully under control as well as the large high voltage required would be inconvenient for operation outside of a laboratory. Therefore, both nuclear emulsions and RPCs were discarded since they do not fulfill all the experimental requirements of the MU-RAY project.

A plastic scintillator muon telescope is a better candidate to be operated on volcano slopes. This technology, widely used since several decades in Particle Physics, was subject to a continuous development. Nowadays, excellent results are obtained by the combined use of plastic scintillator strips and scintillating wavelength-shifting (WLS) fibers running along the strip. The principle relies on the use of a cheap scintillator, with small photon-absorption length, which produces photons that are transported with high efficiency by WLS fibers onto the surface of a photo-multiplier tube (PMT). A plastic-scintillator detector requires a minimal maintenance, has low power consumption and is relatively insensitive to ambient conditions. Detector planes based on plastic scintillators with an area of approximately $50 \mathrm{~m}^{2}$ have been constructed and successfully operated, with detector total areas of thousands of $\mathrm{m}^{2}$.

Successful muon radiographies of volcanoes were obtained by means of plastic scintillator telescopes (Tanaka $e t$ al., 2005; Tanaka and Yokoyama, 2008). They were made of 8 to $10 \mathrm{~cm}$ wide plastic scintillator strips, covering a total area of approximately $1 \mathrm{~m}^{2}$ and providing angular resolutions down to $40 \mathrm{mrad}$. Each strip was read out by a photomultiplier through a light guide.

The outcome of the discussions was that plastic scintillator strips read out by WLS fibers must be considered as the baseline MU-RAY detector technology.

\subsection{The OPERA scintillator strips and readout system}

The neutrino oscillation experiment OPERA (http://www.cern.ch/opera) at the Gran Sasso National Laboratory employs on a large-scale plastic scintillator strips readout by optical WLS fibers and multi-anode PMTs for the Target Tracker (Adam et al., 2007), which predicts in real time the location of the event in the nuclear emulsions to guide their analysis. Figure 1 shows the scintillator strips and the readout electronics of the OPERA experiment. Each strip is $2.6 \mathrm{~cm}$ wide and about $7 \mathrm{~m}$ long. A groove in the strip allocates a WLS optical fiber. The fiber conveys the optical signal to a multi-anode PMT (Hamamatsu H8804MOD1), which reads the signals of 64 strips. A mechanical module thus consists of 64 strips and their readout. Modules are assembled together, to form planes with approximate size of $7 \times 7 \mathrm{~m}^{2}$. Two planes of strips, with horizontal and vertical orientation, are grouped together to predict the event location in each plane of emulsion detector. In total, the plastic scintillator strips cover an area of 2,900 $\mathrm{m}^{2}$. On the average, 6 photoelectrons are detected in the $7 \mathrm{~m}$ long scintillator tiles, for normal incident muons.

The readout electronics is based on a 32-channel ASIC. It includes a fast shaper for triggering purposes and a slow shaper for the measurement of the collected charge. Two chips are used to readout each multi-anode PMT, for a total of 1984 chips for the full detector. A detailed description of the ASIC design and performance can be found in ref. (Lucotte et al., 2004). The DAQ board of the OPERA experiment (Girerd et al., 2000; PATENT, 2006) is a compact data acquisition system able to read and control the signals generated by a multi-anode PMT. It is controlled by an Ethernet network through a LINUX operating system.

Several technical developments stemmed out of the Opera Target Tracker system, of which muon radiography could profit.

Taking into account the pixel effects, two $x-y$ planes made of the $2.6 \mathrm{~cm}$ wide Opera-scintillator strips at $1 \mathrm{~m}$ distance would provide a spatial resolution of approximately $30 \mathrm{mrad}$.

\subsection{The MINERvA plastic scintillator design}

An appealing performance is given by the design of the scintillators of the Inner Detector of the Minerva experiment (http://minerva.fnal.gov/) at FNAL. That detector features planes of scintillators strips of isosceles triangular profile $(3.3 \mathrm{~cm}$ base, $1.7 \mathrm{~cm}$ height). The scintillators are produced at the FNAL-NICADD extrusion facility (Pla-Dalmau et al., 2001, 2003); a polystirene core doped with PPO (1\%) and POPOP $(0.03 \%)$ are co-extruded with 

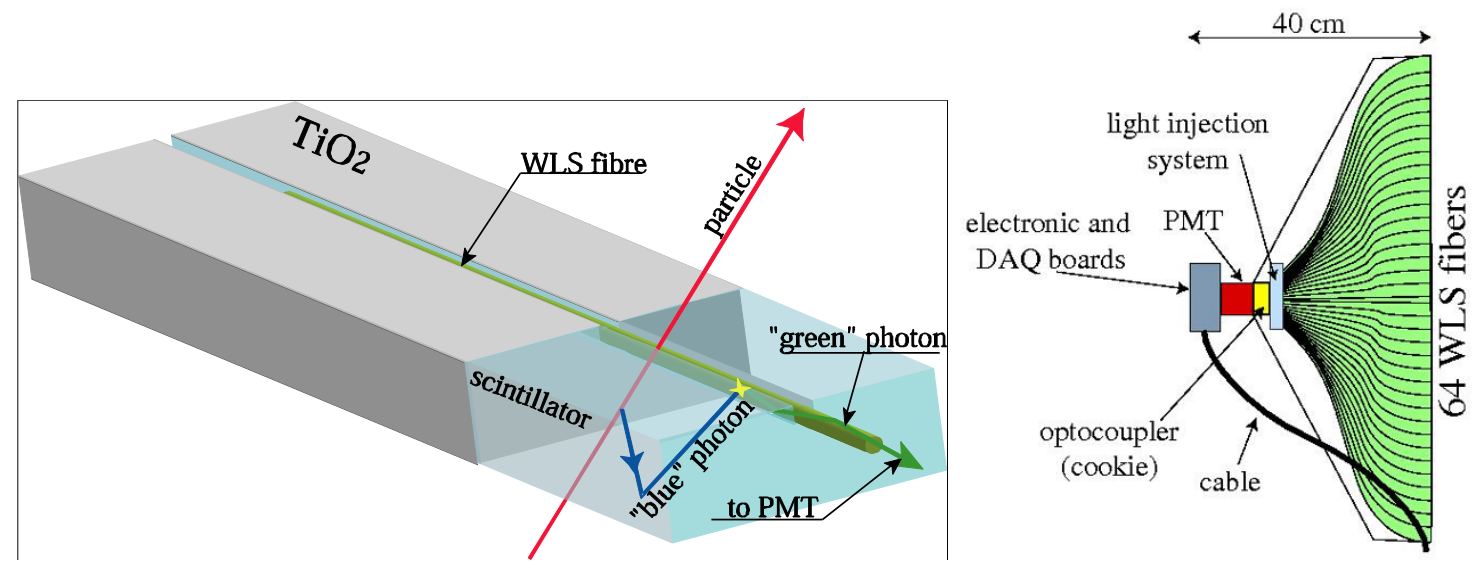

Fig. 1. The plastic scintillator strips (left figure) of the OPERA neutrino experiment and their readout system made by optical wavelength shifting fibres and multianode PMT (right figure).

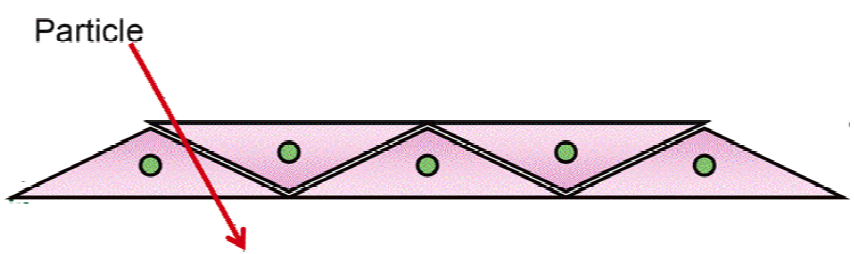

Fig. 2. Schematic drawing of a section of a plane made by the Minerva scintillation bars. The dots represent the WLS fibers running through the scintillator.

$0.25 \mathrm{~mm}$ thick $\mathrm{TiO}_{2}$ reflective coating (Pla-Dalmau et al., 2005). The strips are readout by embedded WLS fibers glued in a $2.6 \mathrm{~mm}$ hole at the centre of the strip. The fibers are Kuraray Y11 S-35 type, 175 ppm of dopant, multicladded with a $1.2 \mathrm{~mm}$ diameter. They are read from one end and the other end is mirrored. The fibers are coupled to multi-anode Hamamatsu photomultipliers. The geometrical assembly of the scintillators, shown in Fig. 2, allows a spatial resolution that is determined by the charge sharing between two adjacent scintillator strips traversed by a particle. Test-beam data did show single hit spatial resolutions below $3 \mathrm{~mm}$; such resolution translates to $\approx 10 \mathrm{mrad}$ for the measurement of two $x-y$ planes positioned at $0.5 \mathrm{~m}$ distance thus significantly improving the spatial resolution obtained with rectangular-profile scintillators of similar transverse dimensions. An average of approximately 20 photoelectrons are detected in two adjacent scintillators when a normal incident m.i.p. crosses a plane.

\subsection{The Silicon Photo-Multiplier option}

Silicon Photo-Multipliers (SiPM) are semiconductor photo-detectors operated in limited-Geiger mode (Buzhan et al., 2001, 2003; Sadygov et al., 2003; Golovin et al., 2004). They are operated at $\sim 50 \mathrm{~V}$ and the power needed per pixel is $\sim 50 \mu \mathrm{W}$. They are also called Multi-Pixel Photon Counters (MPPC) since they typically host 1000 pixels in $1 \mathrm{~mm}^{2}$ connected in series (see Fig. 3); each pixel gain is $\sim 10^{6}$ and gives a signal of $\sim 1 \mathrm{mV}$ for a typical $10-20$ ns pulse length. They have sub-nanosecond time resolution. Their quantum efficiency is $\sim 100 \%$, but factors like geometric-sensitive area, probability for photo-detection to initiate a Geiger discharge and recovery time for the pixel, give an overall photon detection efficiency of 30-70\%, to be compared to a typical value of $15 \%$ of multi-anode PMTs. The excellent single photo-electron resolution allows rather direct calibration and monitoring. SiPM have been introduced in the Particle Physics community since a decade. So far, they were adopted by a few experiments and projects, e.g. the T2K near detector (Gomi et al., 2007) and the CALICE Collaboration for the International Linear Collider, which successfully tested a 8,000 channels prototype of the analogue HCAL prototype calorimeter (Repond et al., 2008). Nowadays, SiPM are claimed to be less expensive than PMTs.

Since this technology is rather recent, there are several caveats to their use. Although the electronic noise is negligible, the single pixel dark noise (given mostly by thermal pairs produced in the semiconductor) is of the order of magnitude of a $\mathrm{MHz}$ at room temperature and increases with increasing temperature or bias voltage. The dark noise could obviously be controlled by raising the threshold to a few pixels or by reducing the bias voltage at the expenses of the gain. However, these procedures must be carefully evaluated and tested in the case of the detection of a m.i.p., like in our case, in which a threshold should be set to a few photoelectrons. A more worrisome problem is given by the inter-pixel optical cross talk and the after-pulses, which are related in time to a firing pixel. These effects are measured at room temperature to give an average of approximately 1.25 pixels firing by one initial photo-electron. New generation SiPMs were recently produced, for which the optical cross talk and the after-pulses were reduced to a several percent (Piemonte et al., 2007).

The use of SiPM would however bring several advantages. Among them: simplification of the optical system by 


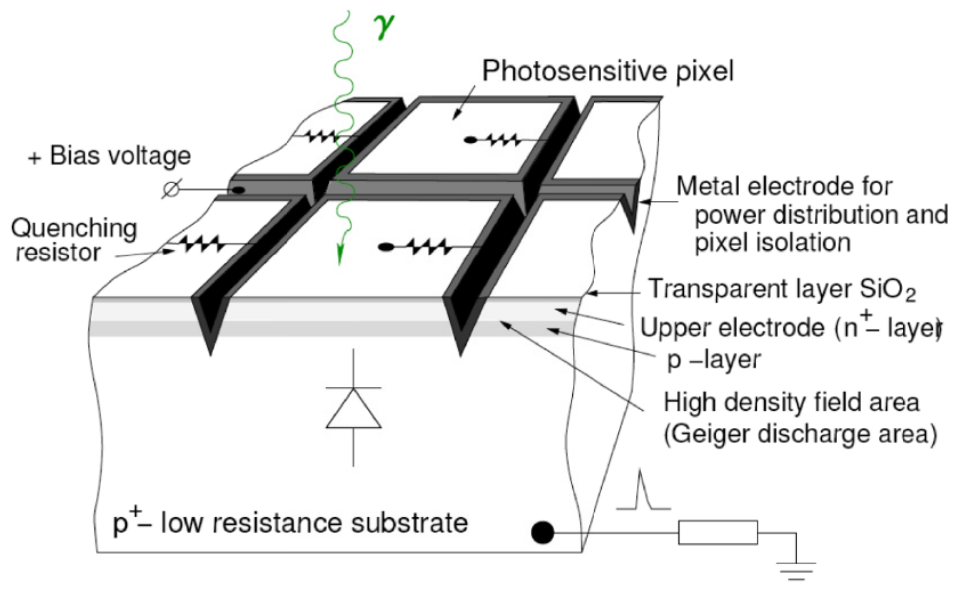

Fig. 3. Schematic view of a SiPM.

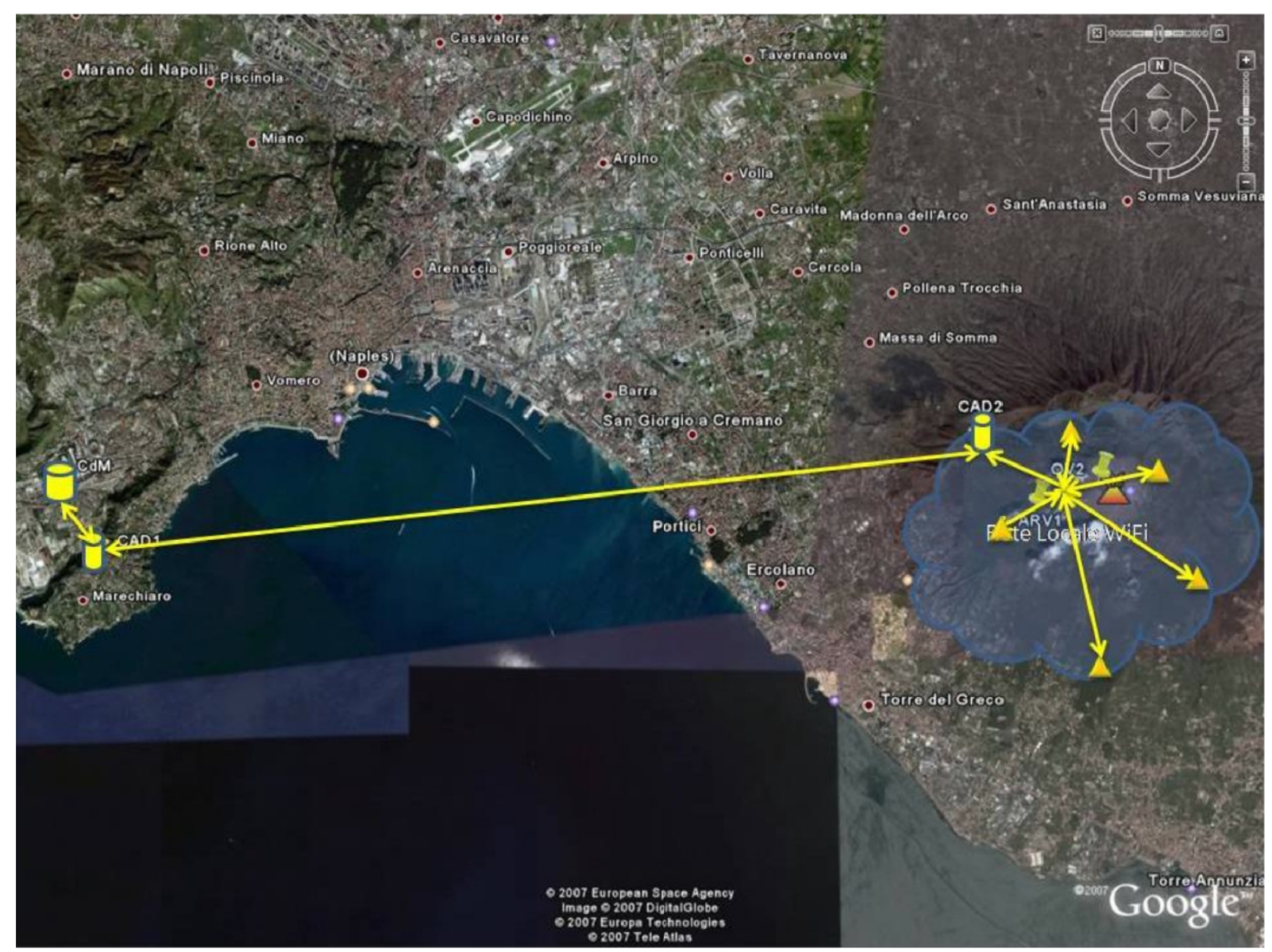

Fig. 4. Map of the Naples gulf area, showing the a schematics of the wireless system of Mt. Vesuvius.

mounting the single channel SiPM directly on the scintillator surface, thus avoiding complex fiber systems; straightforward single channel calibration, owing to the excellent single pixel resolution; increased ruggedness of the system, which will be an advantage in volcanic environments; reduction in cost.

\subsection{Power and data transmission}

Since electrical power lines will not be available on volcano slopes, one has to design a detector that requires very little power. As discussed in the next section, the total power consumption of one MU-RAY telescope is expected to be less than $70 \mathrm{~W}$. Given the favourable solar radiance in the Mt. Vesuvius area, one can use a set of commercially available solar cells. Since it is desirable to have the system running without interruptions to prevent damages to the electronics, one should have a set of batteries to store enough energy to run the detector even in the occurrence of up to seven consecutive cloudy days. The estimated need 


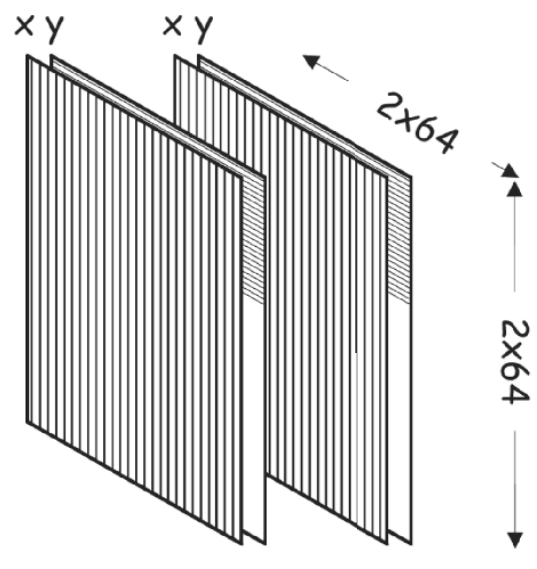

Fig. 5. Schematic drawing showing the strip distribution in the two MU-RAY telescope modules.

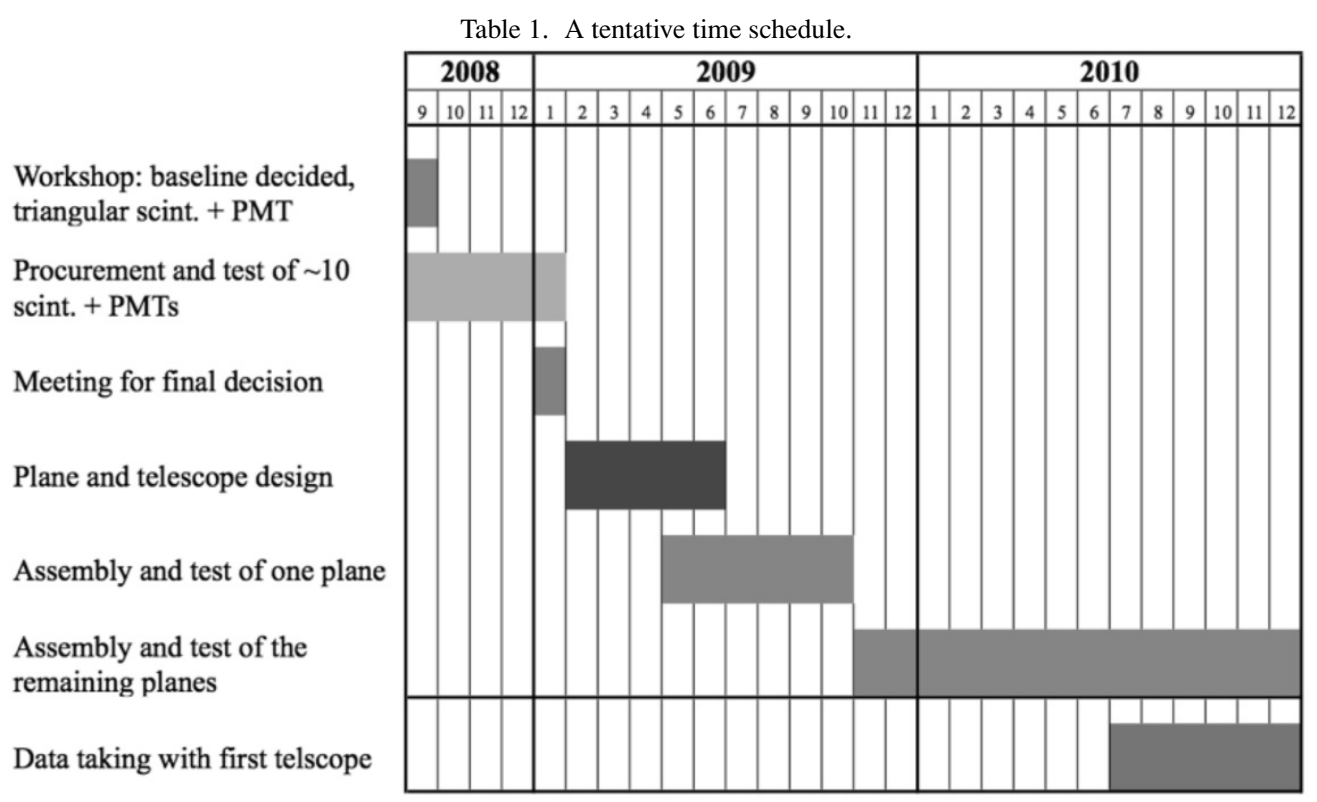

amounts to approximately $200 \mathrm{~A}$ h/day. The usage of a two axis solar tracker to increase efficiency has also been considered.

The MU-RAY project data transmission will profit of the Mt. Vesuvius $5 \mathrm{GHz}$ wireless seismic network of the Vesuvian Observatory shown in Fig. 4 (Scarpato et al., 2008). Currently the infrastructure manages a total of 79 channels with a 24-bit at 100 cps sampling ( $190 \mathrm{kbps})$, but the network has a much greater potential, that will be made available to MU-RAY. Part of the data is collected in local data acquisition centers. Then, the real time signals are centralized at the Monitoring Center of the Vesuvian Observatory. The backbone WiFi link covers about $18 \mathrm{~km}$ in line of sight between the site in Naples at about $200 \mathrm{~m}$ above sea level and the second one on the Mt. Vesuvius, in the historic building of the Vesuvian Observatory at about $600 \mathrm{~m}$ above sea level. Since the distance among the stations and between each station and the data acquisition center varies from hundreds of meters to several kilometers, the data transmission through the wireless network is not expected to limit the choice of the locations of the experimental sites.

\subsection{Baseline detector design}

The use of the MINERvA scintillators and fibers together to the OPERA Target Tracker read-out system was proposed as the baseline technology to be adopted for the construction of the first MU-RAY telescope. According to the experience of Tanaka et al., the combination of two $x-y$ hits will be enough to measure the direction of a muon. Therefore it is foreseen that a MU-RAY telescope, in his minimal version, will be made of two modules (see Fig. 5), each carrying two planes of strips oriented perpendicularly to each other to provide the $x$ and $y$ coordinate of the crossing muon. Each plane will contain 128 two-meter long strips, for a total width of $211.2 \mathrm{~cm}$. Therefore the total number of channels for a complete telescope will be 512, which could be served by eight 64 channel photo-multipliers. The Opera system (64 channels multi-anode PMT, front-end electronics and DAQ) is taken as baseline option. It is expected to absorb approximately $8 \mathrm{~W}$ for 64 channels, which would amount to a total power consumption of less than $70 \mathrm{~W}$ for the entire telescope.

For an easier transportation and handling, each plane will be split in two half planes of $1 \times 2 \mathrm{~m}^{2}$. The aim is to limit 
the weight of each half plane to approximately $50 \mathrm{~kg}$.

During the workshop it was also foreseen to start an R\&D programme to test some of the SiPMs commercially available. Given the limited number of photo-electrons produced in the scintillator by the crossing of a muon, SiPMs with a few hundreds pixels should be enough to guarantee a linear response and avoid saturation effects. Conformity to usage in the volcanic conditions will be established by measurements with cosmic rays or at a test beam.

\section{Tentative Time Schedule}

A tentative time schedule is shown in Table 1. The technical solutions discussed at the workshop will be studied and tested, with the aim of taking the final decisions at the beginning of 2009 . One will then proceed with the final design, to start assembly and test of the first module at mid 2009; if successful, one will continue building the other planes, with the aim of taking data with the first telescope in the second half of 2010. The three MU-RAY telescopes should be completed by the end of 2010. The year 2011 will be devoted to data taking.

\section{References}

Adam, T. et al., The OPERA experiment Target Tracker, Nucl. Instr. Meth., A577, 523, 2007.

Auger, E., P. Gasparini, J. Virieux, and A. Zollo, Seismic evidence of an extended magmatic sill under Mt. Vesuvius, Science, 294, 1510-1513, 2001.

Buontempo, S., L. D’Auria, G. De Lellis, G. Festa, P. Gasparini, G. Iacobucci, A. Marotta, M. Martini, G. Miele, P. Migliozzi, O. Pisanti, P. Strolin, M. Vassallo, and A. Zollo, Perspectives for the radiography of Mt. Vesuvius by cosmic ray muons, Earth Planets Space, 62, this issue, 131-137, 2010.

Buzhan, P. et al., An advanced study of silicon photomultiplier, ICFA Instrum. Bull., 23, 28, 2001.

Buzhan, P. et al., Silicon photomultiplier and its possible applications, Nucl. Instr. Meth., A504, 48, 2003.

Ferrari, A. et al., FLUKA: A multi-particle transport code (Program version 2005), CERN-2005-010, SLAC-R-773, INFN-TC-05-11, Oct 2005, 405 pp, 2005.

GEANT4: a toolkit for the simulation of the passage of particles through matter, http://www.geant4.org/geant4/.

Gibert, D., F. Beauducel, Y. Déclais, N. Lesparre, J. Marteau, F. Nicollin, and A. Tarantola, Muon tomography: Plans for observations in the Lesser Antilles, Earth Planets Space, 62, this issue, 153-165, 2010.

Girerd, C., S. Gardien, J. Burch, S. Katsanevas, and J. Marteau, Ethernet network-based DAQ and smart sensors for the OPERA long-baseline neutrino experiment, Nuclear Science Symposium Conference Record, 2000 IEEE, 2, 12/111-12/115, 2000.

Golovin, V. et al., Novel type of avalanche photodetector with Geiger mode operation, Nucl. Instr. Meth., A518, 560, 2004.

Gomi, S. et al., Development and study of the multi pixeld photon counter, NIM, A581, 427, 2007.

Lucotte, A. et al., A front-end read out chip for the OPERA scintillator tracker, Nucl. Instr. Meth., A521, 378, 2004.

Miele, G. et al., The aperture for UHE tau neutrinos of the Auger fluorescence detector using a digital elevation map, Phys. Lett. B, 634, 137, 2006.

PATENT, Installation de capteurs intelligents pour l'acquisition a haut debit de donnees via le reseau Ethernet, Patent \# 0400468 (delivered with number \# 06/16 dated 2006.04.21), 2006.

Piemonte, C. et al., Development of Silicon Photomultiplies at IRST, talk presented at the 1st Workshop on Photon Detectors for High Energ,y Medical and Space Applications, June 13-14, 2007, Perugia, Italy, 2007.

Pla-Dalmau, A., A. D. Bross, and K. L. Mellott, Low-cost extruded plastic scintillator, Nucl. Instr. Meth., A466, 482-491, 2001.

Pla-Dalmau, A., A. D. Bross, and V. V. Rykalin, Extruding plastic scintillator at Fermilab, FERMILAB-CONF-03-318, Proceedings of the 2003 IEEE Nuclear Science Symposium and Medical Imaging Conference, Portland, Oregon, USA October 16-26, 2003.

Pla-Dalmau, A., A. D. Bross, V. V. Rykalin, and B. M. Wood, Extruded plastic scintillator for MINERvA, FERMILAB-CONF-05-506, Proceedings of the 2005 IEEE Nuclear Science Symposium and Medical Imaging Conference, Puerto Rico, October 23-29, 2005.

Repond, J. et al., Design and electronics commissioning of the physics prototype of a Si-W electromagnetic calorimeter for the international linear collider, CALICE Collaboration, JINST 3:P08001, 2008.

Sadygov, Z. Ya. et al., Super-sensitive avalanche silicon photodiode with surface transfer of charge carriers, Nucl. Instr. Meth., A504, 301, 2003.

Scarpato, G. et al., WiFi data transmission system for monitoring volcanic areas: an example application on Mt. Vesuvius, Poster at the European Geosciences Union General Assembly 2008 (EGU2008), April 13-18, Vienna, Austria, Geophysical Research Abstracts, Vol. 10, EGU2008A-09461, 2008, EGU General Assembly, 2008.

Tanaka, H. K. M. and I. Yokoyama, Muon radiography and deformation analysis of the lava dome formed by the 1944 eruption of Usu, Hokkaido-Contact between high-energy physics and volcano physics, Proc. Jpn. Acad., B84, 107-116, 2008.

Tanaka, H., K. Nagamine, S. N. Nakamura, and K. Ishida, Radiographic measurements of the internal structure of Mt. West Iwate with near horizontal cosmic ray muons and future developments, Nucl. Instr. Meth. Phys. Res., A555, 164-172, 2005.

Tanaka, H. K. M., T. Nakano, S. Takahashi, J. Yoshida, H. Ohshima, T. Maekawa, H. Watanabe, and K. Niwa, Imaging the conduit size of the dome with cosmic ray muons: The structure beneath Showa Shinzan Lava Dome, Japan, Geophys. Res. Lett., 34, L22311, doi:10. 1029/2007GL031389, 2007a.

Tanaka, H. K. M., T. Nakano, S. Takahashi, J. Yoshida, M. Takeo, J. Oikawa, T. Ohminato, Y. Aoki, E. Koyama, H. Tsuji, and K. Niwa, High resolution imaging in the inhomogeneous crust with cosmic ray muon radiography: The density structure below the volcanic crater floor of Mt. Asama, Japan, Earth Planet. Sci. Lett., 263, 104-113, 2007 b.

Tanaka, H. K. M., T. Nakano, S. Takahashi, J. Yoshida, and K. Niwa, Development of an emulsion imaging system for cosmic-ray muon radiography to explore the internal structure of a volcano, Mt. Asama, Nucl. Instr. Meth. Phys. Res., A575, 489-497, 2007c.

Tanaka, H. K. M., T. Nakano, S. Takahashi, J. Yoshida, M. Takeo, J. Oikawa, T. Ohminato, Y. Aoki, E. Koyama, H. Tsuji, H. Ohshima, T. Maekawa, H. Watanabe, and K. Niwa, Radiographic imaging below a volcanic crater floor with cosmic-ray muons, Am. J. Sci., 308, 843-850, 2008.

Zollo, A. et al., Seismic evidence for a low-velocity zone in the upper crust beneath Mount Vesuvius, Science, 274, 592-594, 1996.

Zollo, A. et al., Bayesian estimation of 2-D P-velocity models from active seismic arrival time data: imaging of the shallow structure of Mt. Vesuvius (Southern Italy), Geophys. J. Int., 151, 566-582, 2002.

F. Beauducel, A. Bross, S. Buontempo, L. D’Auria, Y. Déclais, G. De Lellis, G. Festa, P. Gasparini, D. Gibert, K. Hoshina, G. Iacobucci (e-mail: giuseppe.iacobucci@na.infn.it), N. Lesparre, G. Macedonio, A. Marotta, J. Marteau, M. Martini, G. Miele, P. Migliozzi, C. A. Moura, M. Orazi, A. Pla-Dalmau, O. Pisanti, S. Pastor, R. Peluso, P. Rubinov, G. Scarpato, G. Sekhniaidze, P. Strolin, H. Taira, M. Tanaka, H. K. M. Tanaka, A. Tarantola, T. Uchida, M. Vassallo, I. Yokoyama, and A. Zollo 